\title{
RANDOM BASES FOR COPRIME LINEAR GROUPS
}

\author{
HÜLYA DUYAN, ZOLTÁN HALASI, AND KÁROLY PODOSKI
}

\begin{abstract}
The minimal base size $b(G)$ for a permutation group $G$, is a widely studied topic in the permutation group theory. Z. Halasi and K. Podoski 8 proved that $b(G) \leq 2$ for coprime linear groups. Motivated by this result and the probabilistic method used by T. Burness, M.W. Liebeck and A. Shalev, it was asked by L. Pyber 19 that for coprime linear groups $G \leq G L(V)$, whether there exists a constant $c$ such that the probability of that a random $c$-tuple is a base for $G$ tends to 1 as $|V| \rightarrow \infty$. While the answer to this question is negative in general, it is positive under the additional assumption that $G$ is even primitive as a linear group. In this paper, we show that almost all 11-tuples are bases for coprime primitive linear groups.
\end{abstract}

\section{INTRODUCTION}

For a finite permutation group $G$, a subset $B$ of $\Omega$ is called a base for $G$, if the pointwise stabilizer of $B$ in $G$ is trivial. The concept of base plays a fundamental role in the development of the algorithms for permutation groups and these algorithms are significantly faster if the size of the base is small (see the book of Á. Seress [22]). The minimal size of a base for $G$ acting on $\Omega$ is denoted by $b(G)$. L. Pyber ([18]) showed that there exists a universal constant $c>0$ such that almost all subgroups $G$ of $\operatorname{Sym}(n)$ satisfy that $b(G)>c n$.

On the other hand, there are several important group classes where the minimal base size $b(G)$ can be bounded by a fixed constant $c$. Á. Seress [21] showed that $b(G) \leq 4$ for a solvable primitive group $G$. For an almost simple primitive permutation group $G$, a famous conjecture of P. J. Cameron and W. M. Kantor [2] states that there exists an absolute constant such that $b(G) \leq c$ for all non-standard primitive permutation groups $G$. In [3, P. J. Cameron suggested that $c$ can be chosen to 6 apart from the Mathieu group $M_{24}$ with its natural action, where the minimal base size is 7 . The Cameron-Kantor conjecture was proved by M. W. Liebeck and A. Shalev in [15]. However, this was an existence result for $c$, using probabilistic method without yielding any explicit value for this constant. Finally, T. C. Burness, M. W. Liebeck and A. Shalev [1 proved that if $G$ is a finite almost simple group in a primitive faithful non-standard action, then $b(G) \leq 7$, with equality if and only if $\mathrm{G}$ is the Mathieu group $M_{24}$ in its natural action of degree 24 .

2010 Mathematics Subject Classification. 20P05, 20C30.

Key words and phrases. coprime linear group, random base.

The work of the second and third authors on the project leading to this application has received funding from the European Research Council (ERC) under the European Union's Horizon 2020 research and innovation programme (grant agreement No 741420). The second author was also supported by the János Bolyai Research Scholarship of the Hungarian Academy of Sciences and by the National Research, Development and Innovation Office (NKFIH) Grant No. K115799. 
Furthermore, they proved that if $\mathrm{G}$ is a finite almost simple group, and $\Omega$ is a primitive faithful non-standard $G$-set then the probability that a random 6-tuple in $\Omega$ is a base for $\mathrm{G}$ tends to 1 as $|\Omega| \rightarrow \infty$. For a finite vector space $V$, a linear group $G \leq G L(V)$ is called coprime, if $(|G|,|V|)=1$. D. Gluck and K. Magaard [7] proved that for such a group the minimal base size of $G$ is bounded by an absolute constant that is $b(G) \leq 94$. Z. Halasi and K. Podoski [8] improved this result by showing that $b(G) \leq 2$ and this estimation is sharp. Based on this result and the random base result of T. C. Burness, M. W. Liebeck and A. Shalev, L. Pyber [19] asked whether for a coprime linear group $G \leq G L(V)$ there exists an absolute constant $c$ such that a random $c$-tuple in $V$ is a base for $G$ tends to 1 as $|V| \rightarrow \infty$. We answer this question affirmatively by showing that

Theorem 1.1. Let $V$ be a finite vector space and $G \leq G L(V)$ be a coprime primitive linear group, i.e. $(|G|,|V|)=1$. Then the probability that a random 11-tuple in $V$ is a base for $G$ tends to 1 as $|V| \rightarrow \infty$.

In fact, we also give lower bounds for this probability in terms of the base field and the dimension of $V$. Our bounds highly depends on the structure of $G$. As a part of our argument, we give a general structure theorem for maximal coprime primitive linear groups in Theorem 4.1 .

For any positive integer $c$ let us define the probability

$$
P b(c, G, V):=P\left(\text { random } v_{1}, \ldots, v_{c} \in V \text { is a base for } G\right) \text {. }
$$

The main goal of this paper is to prove the following

Theorem 1.2. Let $V$ be an $n$-dimensional vector space over the finite field $\mathbb{F}_{q}$ and let $G \leq G L(V)$ be a coprime primitive linear group. Then for any $c \geq 11$, the probability $\mathrm{Pb}(c, G, V)$ is close to zero if $|V|$ is large enough. More precisely, one of the following holds.

(1) $P b(c, G, V) \geq 1-\frac{3}{q^{\left(\frac{c}{2}-5\right) \sqrt{n}}}$;

(2) There is an $\mathbb{F}_{q}^{k}$ vector space structure on $V$ for some field extension $\mathbb{F}_{q}^{k} \geq \mathbb{F}_{q}$ (possibly $k=1$ ) and a tensor product decomposition $V=V_{1} \otimes_{\mathbb{F}_{q}^{k}} U$ over $\mathbb{F}_{q}^{k}$ with $1 \leq \operatorname{dim}(U)<\operatorname{dim}\left(V_{1}\right) \leq n / k$ such that $G \leq \Gamma L\left(\mathbb{F}_{q}^{k}, n / k\right)$ and $H=$ $G \cap G L\left(\left(\mathbb{F}_{q}^{k}, n / k\right)\right.$ preserves this tensor product decomposition. Furthermore, $H=H_{1} \otimes H_{2}$ with $H_{1} \leq G L\left(V_{1}\right), H_{2} \leq G L(U)$ are absolutely irreducible linear groups, and $S_{1}=\operatorname{Soc}\left(H_{1} / Z\left(H_{1}\right)\right)$ is a non-Abelian simple group.

(a) If $S_{1}$ is not an alternating group, then

$$
P b(c, G, V) \geq 1-\left(\frac{1}{q^{(c-4) \sqrt{\operatorname{dim}(V)}}}+\frac{2}{|V|^{(c-10) / 80}}\right) ;
$$

(b) If $S_{1} \simeq A_{m}$ for some $m$ and $V_{1}$ is not an irreducible component of the natural permutation $\mathbb{F}_{q}^{k} A_{m}$-module, then

$$
P b(c, G, V) \geq 1-\frac{3}{q^{\frac{c-10}{16} \sqrt{\operatorname{dim}(V)}}} ;
$$

(c) If $S_{1} \simeq A_{m}$ for some $m$ and $V_{1}$ is the non-trivial irreducible component of the natural permutation $\mathbb{F}_{q}^{k} A_{m}$-module, then

$$
P b(c, G, V) \geq 1-\frac{3}{n^{c-2}} .
$$


Remark 1.3.

(1) Let $Z=Z(G L(V)) \simeq \mathbb{F}_{q}^{\times}$denote the group of scalar transformations on $V$. If $G \leq G L(V)$ is a coprime linear group on $V$, then so is $G Z \geq G$ and we have $P b(c, G, V) \geq P b(c, G Z, V)$. Therefore, for the rest of this paper we will always assume that $G$ contains $Z$.

(2) The assumption "primitive" is necessary here. To see this, let $H \leq G L(n, q)$ be the group of all invertible diagonal matrices, so $H \simeq\left(\mathbb{F}_{q}^{\times}\right)^{n}$. Then $v_{1}, \ldots, v_{c} \in \mathbb{F}_{q}^{n}$ is a base for $H$ if and only if for each $1 \leq i \leq n$ the $i$-th component of some $v_{j}$ is non-zero. For any fixed $i$, this has probability $\left(1-1 / q^{c}\right)$, so we have

$$
P b\left(c, H, \mathbb{F}_{q}^{n}\right)=\left(1-\frac{1}{q^{c}}\right)^{n},
$$

which is close to zero for any fixed $c$ and big enough $n$. If $(q, n)=1$, then one can add the regular permutation action of $C_{n}$ on the components of $\mathbb{F}_{q}^{n}$ to get the coprime irreducible linear group $G=H \rtimes C_{n} \leq G L(n, q)$ satisfying $\lim _{n \rightarrow \infty} P b\left(c, G, \mathbb{F}_{q}^{n}\right)=0$.

\section{Bounds on $P b(c, G, V)$ in terms of supports and Character ratios}

In order to prove Theorem 1.2 our primary tool will be the concept of support for elements of a linear group.

Definition 2.1. For a linear group $G \leq G L(V)$ and a $g \in G$ the fixed-point space and the support of $g$ are defined as

$$
\operatorname{Fix}(g):=\{v \in V \mid g(v)=v\} \text { and } \operatorname{Supp}(g):=\operatorname{dim}(V)-\operatorname{dim}\left(\operatorname{Fix}_{V}(g)\right) .
$$

Furthermore, let the minimum support of $G$ be defined as

$$
\operatorname{MinSupp}(G):=\min _{1 \neq g \in G} \operatorname{Supp}(g) .
$$

We use the notation $\operatorname{Fix}_{V}(g), \operatorname{Supp}_{V}(g)$ and $\operatorname{MinSupp}_{V}(G)$ if we also want to highlight the vector space on which the group acts.

Remark 2.2. If $G$ strictly contains $Z$, then $\operatorname{MinSupp}(G)$ equals

$$
\min _{g \in G \backslash Z}\left(\operatorname{dim}(V)-\max _{\lambda \in \mathbb{F}_{q}^{\times}}\left(\operatorname{dim}\left(\operatorname{ker}\left(g-\lambda \cdot \operatorname{id}_{V}\right)\right)\right)\right) .
$$

In order to get bounds for $\operatorname{MinSupp}_{V}(G)$ in case of $G \leq G L(V)$ is a quasisimple coprime linear group, we will use results from character ratios of complex irreducible characters of such groups.

Definition 2.3. For a finite group $G$ and $\chi \in \operatorname{Irr}(G)$ with $\chi(1) \neq 1$ let us define the maximal character ratios

$$
\operatorname{mr}(G, \chi):=\max _{g \notin Z(\chi)} \frac{|\chi(g)|}{\chi(1)} \text { and } \operatorname{mr}(G):=\max _{\chi \in \operatorname{Irr}(G), \chi(1) \neq 1} \operatorname{mr}(G, \chi) .
$$

Clearly, $\operatorname{mr}(G)<1$ for every finite group $G$.

The connection between minimal support and maximal character ratio is described in the following Lemma. 
Lemma 2.4. Let $V$ be an n-dimensional vector space over the finite field $\mathbb{F}_{q}$ and let $G \leq G L(V)$ be a non-Abelian coprime irreducible linear group. Then we have

$$
\operatorname{MinSupp}_{V}(G) \geq \frac{\operatorname{dim}(V)}{2}(1-\operatorname{mr}(G)) .
$$

Moreover, if $\chi \in \operatorname{Irr}(G)$ is any irreducible component of the Brauer character associated to $V$, then

$$
\operatorname{MinSupp}_{V}(G) \geq \frac{1}{2}\left(\chi(1)-\max _{g \notin Z(\chi)}|\chi(g)|\right) .
$$

Proof. Let $\overline{\mathbb{F}}_{q}$ be the algebraic closure of $\mathbb{F}_{q}$ and let $\bar{V}=V \otimes \overline{\mathbb{F}}_{q}$ be the $\overline{\mathbb{F}}_{q} G$-module arising from the $\mathbb{F}_{q} G$-module $V$. Let $\bar{V}=V_{1} \oplus \ldots \oplus V_{t}$ be the decomposition of $\bar{V}$ into irreducible $\overline{\mathbb{F}}_{q} G$-modules. Then the corresponding representations $G \mapsto$ $G L\left(V_{i}\right)$ form a single Galois conjugacy class by [10, Theorem 9.21], so $\operatorname{Supp}_{V_{i}}(g)=$ $\frac{1}{t} \operatorname{Supp}_{V}(g)$ holds for every $g \in G$. Let $\chi_{i}: G \mapsto \mathbb{C}$ be the irreducible Brauer character associated to $V_{i}$ for each $1 \leq i \leq t$. Since $(q,|G|)=1$, we get $\chi_{i} \in \operatorname{Irr}(G)$ by [10, Theorem 15.13]. Furthermore,

$$
\chi_{i}(1)=\operatorname{dim}\left(V_{i}\right) \quad \text { and } \quad\left[\chi_{i}\langle g\rangle, 1_{\langle g\rangle}\right]=\operatorname{dim}\left(\operatorname{Fix}_{V_{i}}(g)\right) .
$$

For any $g \in G$ we have $\chi_{1}(g)=\left(\chi_{1}(1)-k\right) \cdot 1+\varepsilon_{1}+\ldots+\varepsilon_{k}$ where $k=\operatorname{Supp}_{V_{1}}(g)$ and $\varepsilon_{1}, \ldots, \varepsilon_{k}$ are $o(g)$-th root of unity. Then $\left|\chi_{1}(g)\right| \geq \chi_{1}(1)-2 k=\chi_{1}(1)-$ $2 \operatorname{Supp}_{V_{1}}(g)$ holds, so $2 \operatorname{MinSupp}_{V_{1}}(G) \geq \chi_{1}(1)-\max _{g \notin Z\left(\chi_{1}\right)}\left|\chi_{1}(g)\right|$. (Note that the assumption that $G$ is non-Abelian implies that the $\chi_{i}$ are non-linear characters. Furthemore, if $1 \neq g \in Z\left(\chi_{1}\right)$, then $\operatorname{Supp}_{V}(g)=\operatorname{dim}(V)$, so $\operatorname{MinSupp}_{V}(G)=$ $\min _{g \notin Z\left(\chi_{1}\right)} \operatorname{Supp}_{V}(g)$ must hold.)

It follows that

$$
\begin{aligned}
2 \operatorname{MinSupp}_{V}(G) & =2 t \operatorname{MinSupp}_{V_{1}}(G) \geq t\left(\chi_{1}(1)-\max _{g \notin Z\left(\chi_{1}\right)}\left|\chi_{1}(g)\right|\right) \\
& =t \chi_{1}(1)\left(1-\operatorname{mr}\left(G, \chi_{1}\right)\right) \geq \operatorname{dim}(V)(1-\operatorname{mr}(G)) .
\end{aligned}
$$

Now, the first inequality proves the second claim, while the second inequality proves the first claim.

\section{Lemma 2.5.}

$$
P b(c, G, V) \geq 1-\sum_{1 \neq g \in G} \frac{1}{q^{c \cdot \operatorname{Supp}(g)}} \geq 1-\frac{|G|}{q^{c \cdot \operatorname{MinSupp}(G)}} \geq 1-\frac{1}{|V|^{c(1-\operatorname{mr}(G)) / 2-2}} .
$$

In particular, $\mathrm{Pb}(c, G, V) \geq 1-\frac{1}{|V|^{\varepsilon}}$ for $c \geq \frac{4+2 \varepsilon}{1-\operatorname{mr}(G)}$.

Proof.

$$
\begin{aligned}
& P\left(\left\{v_{1}, \ldots, v_{c}\right\} \subseteq V \text { is not a base for } G\right) \leq \sum_{1 \neq g \in G} P\left(g\left(v_{i}\right)=v_{i}, \forall 1 \leq i \leq c\right) \\
& =\sum_{1 \neq g \in G}\left(\frac{|\operatorname{Fix}(g)|}{|V|}\right)^{c}=\sum_{1 \neq g \in G} \frac{1}{q^{c \cdot \operatorname{Supp}(g)}} \leq \frac{|G|}{q^{c \cdot \operatorname{MinSupp}(G)}} \\
& \leq \frac{|V|^{2}}{\left(q^{n}\right)^{c(1-\operatorname{mr}(G)) / 2}}=\frac{1}{|V|^{c(1-\operatorname{mr}(G)) / 2-2}},
\end{aligned}
$$

and the claim follows. 


\section{BOUNDS FOR CHARACTER RATIOS AND FOR MINIMAL SUPPORTS OF QUASISIMPLE LINEAR GROUPS}

The goal of this section is to give lower bounds for minimal supports of coprime quasisimple groups $G \leq G L(V)$ in terms of $|G|$ and $\operatorname{dim}(V)$.

First we handle the case when $G$ is a sporadic group or a finite quasisimple group of Lie type. For such groups, we use bounds for their maximal character ratios $\operatorname{mr}(G)$.

Theorem 3.1. Let $G$ be a finite quasisimple group such that $G / Z(G)$ is not an alternating group.

(1) If $G / Z(G)$ is a sporadic simple group, then $\operatorname{mr}(G)<0.54$.

(2) If $G=G(r)$ is a finite quasisimple group of Lie type over the field $\mathbb{F}_{r}$, then

$$
\operatorname{mr}(G) \leq \begin{cases}\max \left(\frac{1}{\sqrt{r}-1}, \frac{9}{r}\right) & \text { if } r>9 \\ \frac{19}{20} & \text { if } r \leq 9 .\end{cases}
$$

Proof. We checked part (1) for the covering groups of the sporadic simple groups by using the GAP [4] Character table library and also the undeposited GAP package FUtil to turn cyclotomic complex numbers into floating ones in order to be able to compare the values of $|\chi(g)|$ for various $g$ and $\chi$.

Regarding part (2), it is a simplified version of a result of Gluck 6]. (For a summary of his results, see also [14, Theorem 2.4]).

Remark 3.2. For simple groups of alternating type there is no general upper bound for $\operatorname{mr}(G)$ smaller than 1 . Moreover it can be shown that for every $\varepsilon>0$, the number of irreducible characters $\chi \in \operatorname{Irr}\left(S_{m}\right)$ (or $\left.\chi \in \operatorname{Irr}\left(A_{m}\right)\right)$ satisfying $\operatorname{mr}\left(S_{m}, \chi\right)>1-\varepsilon$ is not bounded if $m$ is large enough.

Corollary 3.3. Let $V$ be a vector space over the finite field $\mathbb{F}_{q}$ and let $G=Z \cdot G_{0} \leq$ $G L(V)$ where $G_{0}$ is a coprime quasisimple irreducible linear group which is not of alternating type. Then $\operatorname{MinSupp}_{V}(G) \geq \frac{1}{40} \operatorname{dim}(V)$.

Proof. By Theorem 3.1, we have $\operatorname{mr}(G)=\operatorname{mr}\left(G_{0}\right) \leq \frac{19}{20}$, so the claim follows from Lemma 2.4.

Now, we handle the case when $\operatorname{Soc}(G / Z(G))$ is an alternating group.

Theorem 3.4. Let $G=S_{m}$ and $\chi=\chi^{(\lambda)} \in \operatorname{Irr}(G)$ corresponding to the partition $\lambda=\left(\lambda_{1} \geq \ldots \geq \lambda_{k}\right)$ of $[m]$. Then $\chi^{\lambda}(1)-\chi^{\lambda}((123)) \geq \frac{1}{m-1} \chi^{\lambda}(1)$ unless

$$
\lambda \in\{(m) ;(1, \ldots, 1)\}
$$

Proof. First, we introduce some notation. Let $\lambda=\left(\lambda_{1} \geq \ldots \geq \lambda_{k}\right)$ be a partition of $m$, different from the two exceptional ones given in the theorem. For any natural numbers $i_{1}, \ldots, i_{k}$ let $\chi^{\lambda-\left\{i_{1}, \ldots, i_{k}\right\}}$ be the character of $S_{m-k}$ corresponding to the Young diagram obtained from the diagram of $\lambda$ by deleting the last cells of the $i_{1}$-th $, \ldots, i_{k}$-th row in that order with the assumption that $\lambda-\left\{i_{1}, \ldots, i_{s}\right\}$ is a valid Young diagram for each $1 \leq s \leq k$. Otherwise, we define $\chi^{\lambda-\left\{i_{1}, \ldots, i_{k}\right\}}$ as the constant zero function on $S_{m-k}$. 
By the Murnaghan-Nakayama rule (see [11, 21.1]),

$$
\begin{aligned}
\chi^{\lambda}((123)) & \leq \sum_{\nu \in\{\lambda-r h(3)\}} \chi^{\nu}(1)+\sum_{\nu \in\{\lambda-r h(1,1,1)\}} \chi^{\nu}(1) \\
& =\sum_{\nu \in\{\lambda-r h(3)\}} \chi^{\nu}(1)+\sum_{\nu \in\{\bar{\lambda}-r h(3)\}} \chi^{\nu}(1)
\end{aligned}
$$

where $\{\lambda-r h(*)\}$ denotes the set of partitions of $m-3$ which we can get from the Young-diagram of $\lambda$ be removing a rim 3-hook of type $(*)$ such that the remaining cells form a valid Young diagram.

On the other hand, by using the branching rule (three times) one gets

$$
\chi^{\lambda}(1)=\sum_{i, j, k} \chi^{\lambda-\{i, j, k\}}(1) .
$$

Let $\nu \in\{\lambda-r h(3)\}$. Then $\nu=\lambda-\{i, i, i\}$ for some (unique) $i$. Now, there is a $j \neq i$ such that $\tau=\lambda-\{i, i, j\}$ is a valid Young diagram. Then both induced characters $\left(\chi^{\tau}\right)^{S_{m-2}}$ and $\left(\chi^{\nu}\right)^{S_{m-2}}$ contain $\chi^{\lambda-\{i, i\}}$ as a component which results $\chi^{\nu}(1) \leq \chi^{\lambda-\{i, i\}}(1) \leq(m-2) \chi^{\tau}(1)$. The same argument can be applied to any $\nu \in\{\bar{\lambda}-r h(3)\}$. It follows that

$$
\begin{aligned}
\chi^{\lambda}(1) & \geq \sum_{i} \chi^{\lambda-\{i, i, i\}}(1)\left(1+\frac{1}{m-2}\right)+\sum_{i} \chi^{\bar{\lambda}-\{i, i, i\}}(1)\left(1+\frac{1}{m-2}\right) \\
& \geq \frac{m-1}{m-2} \chi^{\lambda}((123)) .
\end{aligned}
$$

Hence $\chi^{\lambda}(1)-\chi^{\lambda}((123)) \geq \frac{1}{m-1} \chi^{\lambda}(1)$ which proves the claim.

This result will be adequate for our purposes only if the degree of $\chi$ is large enough. In order to get an overall picture about the form of Young diagrams defining characters of small degree, we will use a result of Rasala [20. In what follows, we use the terminology from Rasala's paper. For any partition $\lambda$ of $m$, let $|\lambda|=m$ be the order of $\lambda$ and let $\lambda^{*}$ be the partition dual to $\lambda$. The partition $\lambda$ is called primary, if $\lambda \geq \lambda^{*}$, where $\geq$ denotes the standard ordering on partitions. If $\lambda=\left(\lambda_{1} \geq \ldots \geq \lambda_{k}\right)$ is a partition of $k$ and $m \geq \lambda_{1}+k$, then let $m / \lambda$ denote the partition of $m$ defined as $m / \lambda=\left(m-k \geq \lambda_{1} \geq \ldots \geq \lambda_{k}\right)$ and let $\varphi_{\lambda}(m):=\chi^{m / \lambda}(1)$ be the degree of the character of $S_{m}$ associated to $m / \lambda$. (Note that $\varphi_{\lambda}(m)$ is a polynomial in $m$ by [20, Theorem A].) For any set $P$ of partitions of $k$ and for $m$ large enough, let $L(P, m):=\left\{\varphi_{\lambda}(m) \mid \lambda \in P\right\}$ and let $\delta(P, m)$ be the largest degree in $L(P, m)$. Then $P$ is said to be $m$-minimal, if for every primary partition $\mu$ of $m$ either $\chi^{\mu}(1)>\delta(L, P)$ or $\mu=m / \lambda$ for some $\lambda \in P$.

By [20, Main Theorem 1.] (for $k=3$ ) we have

Theorem 3.5. Let $P_{3}$ be the set of all partitions of order at most 3 , that is, $P_{3}=$ $\{\emptyset ;(1) ;(2) ;(1,1) ;(3) ;(2,1) ;(1,1,1)\}$. Then $P_{3}$ is $m$-minimal for every $m \geq 15$.

Thus, by using the hook length formula and the Murnaghan-Nakayama rule we can calculate the exact values of $\chi^{\lambda}(1)$ and $\chi^{\lambda}((123))$ when $\chi^{\lambda}(1)$ is among the first seven smallest character degrees of $S_{m}$ for $m \geq 15$. Otherwise, we get a reasonably large lower bound for $\chi^{\lambda}(1)$. (Note that $\lambda$ or $\lambda^{*}$ is primary and $\chi^{\lambda}(1)=\chi^{\lambda^{*}}(1), \chi^{\lambda}((123))=\chi^{\lambda^{*}}((123))$ holds for every partition $\lambda$ of $m$.) 
Corollary 3.6. Let $\lambda$ be a partition of $m$ for $m \geq 15$ and let $\chi^{\lambda} \in \operatorname{Irr}\left(S_{m}\right)$ be the character of $S_{m}$ associated to $\lambda$. Then $\chi^{\lambda}(1)$ and $\chi^{\lambda}((123))$ are as given in Table [1 or $\chi^{\lambda}(1)>\frac{1}{3} m(m-2)(m-4)$.

\begin{tabular}{lll}
\hline$\lambda$ or $\lambda^{*}$ & $\chi^{\lambda}(1)=\chi^{\lambda^{*}}(1)$ & $\chi^{\lambda}((123))=\chi^{\lambda^{*}}((123))$ \\
\hline$(m)$ & 1 & 1 \\
$(m-1,1)$ & $m-1$ & $m-4$ \\
$(m-2,2)$ & $\frac{1}{2} m(m-3)$ & $\frac{1}{2}(m-3)(m-6)$ \\
$(m-2,1,1)$ & $\frac{1}{2}(m-1)(m-2)$ & $\frac{1}{2}(m-4)(m-5)$ \\
$(m-3,3)$ & $\frac{1}{6} m(m-1)(m-5)$ & $\frac{1}{6}(m-3)(m-4)(m-8)+1$ \\
$(m-3,2,1)$ & $\frac{1}{3} m(m-2)(m-4)$ & $\frac{1}{3}(m-3)(m-5)(m-7)-1$ \\
$(m-3,1,1,1)$ & $\frac{1}{6}(m-1)(m-2)(m-3)$ & $\frac{1}{6}(m-4)(m-5)(m-6)+1$ \\
\hline
\end{tabular}

TABLE 1. Character values of $S_{m}$ when the degree is small.

Now, we give an analogue of Corollary 3.3 for alternating-type groups.

Corollary 3.7. Let $V$ be a vector space over the finite field $\mathbb{F}_{q}$ and let $G=Z \cdot G_{0} \leq$ $G L(V)$ where $G_{0}$ is a coprime irreducible linear group and $G_{0} / Z\left(G_{0}\right) \simeq A_{m}$ for some $m \geq 5$. Let us assume that $V$ is not a component of the natural permutation $\mathbb{F}_{q} A_{m}$-module. Then $\operatorname{MinSupp}_{V}(G) \geq \frac{1}{16} \sqrt{\operatorname{dim}(V)}$.

Proof. As in the proof of Lemma 2.4. $\operatorname{MinSupp}_{V}(G)=t \cdot \operatorname{MinSupp}_{V_{1}}(G)$ and $\operatorname{dim}(V)=t \cdot \operatorname{dim}\left(V_{1}\right)$ where $V_{1}$ is an (absolutely) irreducible component of $\overline{\mathbb{F}}_{q} G$ module $V \otimes \overline{\mathbb{F}}_{q}$. Then the claim clearly follows if we prove that $\operatorname{MinSupp}_{V_{1}}(G) \geq$ $\frac{1}{16} \sqrt{\operatorname{dim}\left(V_{1}\right)}$. In other words, we can assume that $V$ is absolutely irreducible. First let us assume that $G_{0} \simeq A_{m}$ for some $m \geq 9$. Let $\varphi \in \operatorname{Irr}\left(A_{m}\right)$ be the Brauer character associated to $V$ and $\chi \in \operatorname{Irr}\left(S_{m}\right)$ above $\varphi$, i.e. $\left[\chi_{A_{m}}, \varphi\right] \neq 0$. Then either $\chi_{A_{m}}=\varphi$ (if $\chi$ is not self-dual) or $\chi_{A_{m}}=\varphi+\varphi^{(12)}$ (if $\chi$ is self-dual). In the latter case $\varphi((123))=\chi((123)) / 2$, since the conjugacy class $(123)^{S_{m}}$ does not split in $A_{m}$. Let $\epsilon$ be 1 or $1 / 2$ according to these cases, so $\varphi(1)=\epsilon \chi(1)$ and $\varphi((123))=\epsilon \chi((123))$. By [20, Result 2.], we have $\operatorname{dim}(V)=\epsilon \chi(1) \geq \frac{1}{2} m(m-3)$. If $\varphi((123))<0$, then $\operatorname{Supp}_{V}((123)) \geq \frac{1}{2} \operatorname{dim}(V) \geq \frac{1}{4} \sqrt{\operatorname{dim}(V)}$ holds trivially. Otherwise, by using Lemma 2.4 and Theorem 3.4 we get that

$$
\begin{aligned}
\operatorname{Supp}_{V}((123)) & \geq \frac{1}{2}(\varphi(1)-|\varphi((123))|)=\frac{\epsilon}{2}(\chi(1)-\chi((123)))=\frac{\epsilon \chi(1)}{2(m-1)} \\
& =\frac{\operatorname{dim}(V)}{2(m-1)} \geq \frac{\sqrt{m(m-3) / 2} \sqrt{\operatorname{dim}(V)}}{2(m-1)} \geq \frac{1}{4} \sqrt{\operatorname{dim}(V)} .
\end{aligned}
$$

For any element $1 \neq g \in A_{m}$ there are $x, y \in A_{m}$ such that $[g, x, y]$ is a threecycle. Applying Lemma 4.2 twice, we get that $\operatorname{Supp}_{V}(g) \geq \frac{1}{4} \operatorname{Supp}_{V}((123)) \geq$ $\frac{1}{16} \sqrt{\operatorname{dim}(V)}$.

Now, let us assume that $m>7$ and $G_{0}$ is the universal covering group of $A_{m}$, so $G_{0} \simeq 2 . A_{m}$. Let $z \in G_{0}$ be the generator of $Z\left(G_{0}\right) \simeq C_{2}$ and let $\bar{g} \in A_{m}$ denote 
the image of any $g \in G_{0}$ under the natural surjection by $G_{0} \mapsto A_{m}$. Then $z$ acts on $V$ as a scalar transformation $z(v)=-v$ for all $v \in V$, so $\operatorname{Supp}_{V}(z)=\operatorname{dim}(V)$. Let $t \in G_{0}$ such that $\bar{t}=(12)(34)$. By Theorem [9, Theorem 3.9], $t$ and $t z$ are conjugate, so $z=[h, t]$ for some $h \in G_{0}$. It follows that $\operatorname{Supp}_{V}(t) \geq \frac{1}{2} \operatorname{Supp}_{V}(z)=$ $\frac{\operatorname{dim}(V)}{2}$ by Lemma 4.2. (In fact, by using this argument to $t z$ instead of $t$ one can prove equality here.) Now, for any $g \in G_{0} \backslash Z$ one can choose $x, y \in G$ such that $[\bar{g}, \bar{x}, \bar{y}]$ is conjugate to $\bar{t}$. Using again Lemma 4.2 twice, we get that $\operatorname{Supp}_{V}(g) \geq \frac{1}{4} \operatorname{Supp}_{V}(t)=\frac{1}{8} \operatorname{dim}(V) \geq \frac{1}{16} \sqrt{\operatorname{dim}(V)}$.

For the remaining cases, $\operatorname{dim}(V) \leq \sqrt{\left|G_{0}\right|}<16^{2}$, so $\frac{1}{16} \sqrt{\operatorname{dim}(V)}<1 \leq$ $\operatorname{MinSupp}_{V}(G)$ follows.

The next result gives a bound to the order of most coprime quasisimple linear groups similar to that of $|G| \leq|V|^{2}=q^{2 \operatorname{dim}(V)}$ but using the minimal support $\operatorname{MinSupp}_{V}(G)$ instead of $\operatorname{dim}(V)$.

Theorem 3.8. Let $V$ be a vector space over the finite field $\mathbb{F}_{q}$ and let $G=Z \cdot G_{0} \leq$ $G L(V)$ where $G_{0}$ is a coprime quasisimple irreducible linear group.

Then one of the following holds:

(1) $\log _{q}|G| \leq d \cdot \operatorname{MinSupp}_{V}(G)$ with $d=5$.

(2) $G_{0} \simeq A_{m}$ and $V$ is the non-trivial irreducible component of the natural permutation module of $A_{m}$ over $\mathbb{F}_{q}$.

(3) $G_{0}=G_{0}(r)$ is a finite quasisimple group of Lie type over the finite field $\mathbb{F}_{r}$ with $r \leq 43$, and $|V|$ is bounded by an absolute constant.

Proof. For any sporadic group $S$, let $\widehat{S}$ be its universal covering group and let $q(S)$ be the smallest prime not dividing the order of $S$. By using GAP [4, we checked that for every $\chi \in \operatorname{Irr}(\widehat{S})$, the inequlity

$$
\log _{q(S)}|\widehat{S}|<d \cdot\left(\chi(1)-\max _{g \in \widehat{S}-Z(\chi)}|\chi(g)|\right) / 2
$$

holds with $d>4.22$. (The largest value is attained for 2.J. $J_{2}$ ) Now, if $G \leq G L(V)$ is any finite quasisimple group with sporadic simple quotient $S=G / Z(G)$, then $G$ is a homomorphic image of $\widehat{S}$, and we can view $V$ as an irreducible $\mathbb{F}_{q} \widehat{S}$-module (where $q \geq q(S))$. Now, if $\chi \in \operatorname{Irr}(\widehat{S})$ is any irreducible component of the Brauer character corresponding to $V \otimes \overline{\mathbb{F}}_{q}$, then

$$
\begin{aligned}
\log _{q}|G| & \leq \log _{q(S)}|\widehat{S}|<d \cdot\left(\chi(1)-\max _{g \in \widehat{S}-Z(\chi)}|\chi(g)|\right) / 2 \\
& \leq d \cdot \operatorname{MinSupp}_{V}(\widehat{S}) \leq d \cdot \operatorname{MinSupp}_{V}(G)
\end{aligned}
$$

also holds with $d>4.22$ by Lemma 2.4 .

Next, let $G \simeq A_{m}$ for some $m \geq 15$. Then we have $m<q$ by the coprime assumption. Let us assume that $V$ is not a component of the natural permutation $\mathbb{F}_{q} A_{m}$-module. Let $\varphi \in \operatorname{Irr}\left(A_{m}\right)$ be an irreducible component of the Brauer character associated to $V$ and $\chi \in \operatorname{Irr}\left(S_{m}\right)$ above $\varphi$. By the proof of Corollary [3.7, we have $\varphi(1)=\varepsilon \chi(1)$, and $\varphi((123))=\varepsilon \chi((123))$, where $\varepsilon$ is $1 / 2$ or 1 if $\chi$ is self-dual or not.

If $\chi$ is one of the characters given in Table 1, then $\chi$ is not self-dual. In that case we have

$$
\operatorname{Supp}_{V}((123)) \geq \frac{1}{2}(\chi(1)-|\chi(123)|) \geq \frac{3}{2}(m-3)
$$


by using Lemma 2.4 and the last five rows of Table 1. Otherwise, $\chi(1)>\frac{1}{3} m(m-$ $2)(m-4)$, so we have

$$
\begin{aligned}
\operatorname{Supp}_{V}((123)) & \geq \frac{1}{2}(\varphi(1)-|\varphi(123)|) \geq \frac{1}{4}(\chi(1)-|\chi(123)|) \\
& \geq \frac{\chi(1)}{4(m-1)}>\frac{m(m-2)(m-4)}{12(m-1)} \geq m-3
\end{aligned}
$$

holds if $\varphi(123) \geq 0$. However, if $\varphi(123)<0$, then $\operatorname{Supp}_{V}((123)) \geq \frac{1}{2} \operatorname{dim}(V) \geq$ $m-3$ holds trivially. Thus, $\operatorname{Supp}_{V}((123)) \geq m-3$ holds in any case. Now, for any element $1 \neq g \in A_{m}$ there are $x, y \in A_{m}$ such that $[g, x, y]$ is a three-cycle. Applying Lemma 4.2 twice, we get that $\operatorname{Supp}_{V}(g) \geq \frac{1}{4} \operatorname{Supp}_{V}((123)) \geq \frac{m-3}{4}$ holds for any $1 \neq g \in A_{m}$. Thus, $d \cdot \operatorname{MinSupp}_{V}(G) \geq \frac{d(m-3)}{4} \geq m \geq \log _{m}(m !) \geq \log _{q}|G|$ holds for $d \geq 5$.

Now, let $m \geq 12$ and let $G_{0}$ be the universal covering group of $A_{m}$, so $G_{0} \simeq 2 . A_{m}$. By the proof of Corollary 3.7 we have $\operatorname{MinSupp}_{V}(G) \geq \frac{1}{8} \operatorname{dim}(V)$. Using [13, Main Theorem] we get that

$$
\begin{aligned}
d \cdot \operatorname{MinSupp}_{V}(G) & \geq \frac{d}{8} \operatorname{dim}(V) \geq \frac{d}{8} \min \{\chi(1) \mid \chi \in \operatorname{Irr}(G), \chi(z) \neq \chi(1)\} \\
& \geq d \cdot 2^{\lfloor m / 2\rfloor-4} \geq m \geq \log _{q}|G|
\end{aligned}
$$

holds for $d \geq 3.25$. For the remaining members of Alternating groups and their covers (i.e for $\left.A_{m}, 12 \leq m \leq 14\right)$, for $2 . A_{m}(m=5$ or $8 \leq m \leq 11$ ) and for 6. $A_{6}, 6 . A_{7}$ we used the same algorithm as for sporadic groups.

Finally, let $G_{0}=G_{0}(r)$ be a quasisimple group of Lie type over a finite field $\mathbb{F}_{r}$ with $(r, q)=1$. First, suppose that $r \geq 47$. By part (2) of Theorem 3.1 we have

$$
\operatorname{mr}(G) \leq \max \left(\frac{1}{\sqrt{r}-1}, \frac{9}{r}\right)<\frac{1}{5} .
$$

By using [17, Theorem 1.] and Lemma 2.4.

$$
\log _{q}|G| \leq 2 n=5 \cdot \frac{2 n}{5} \leq 5 \cdot \operatorname{MinSupp}_{V}(G)
$$

For the rest of the proof, suppose that $r \leq 43$. Since $\chi(1)-2 \operatorname{Supp}(g) \leq|\chi(g)|$ for any $\chi \in \operatorname{Irr}(G)$, we have that

$$
\frac{1}{2} \chi(1)\left(1-\frac{|\chi(g)|}{\chi(1)}\right) \leq \operatorname{Supp}(g) .
$$

Using that $\operatorname{mr}(G) \leq \frac{19}{20}$ also holds for all quasisimple groups of Lie-type by part (2) of Theorem 3.1. we obtain that

$$
\frac{\operatorname{dim}(V)}{8} \leq 5 \operatorname{MinSupp}_{V}(G)
$$

by using Lemma 2.4 again. Since (see [12, Table 5.3.A]) $\operatorname{dim}(V) \geq r^{\mathcal{O}(m)}$ (where $m$ denotes the rank of $\left.G_{0}(r)\right)$ and $\log _{q}|G|=\mathcal{O}\left(m^{2} \log r\right)$, there exist only finitely many possible pairs $(m, r)$ such that $\log _{q}|G|>5 \operatorname{MinSupp}_{V}(G)$. Furthermore, for any fixed $(m, r)$, the inequality $\log _{q}|G| \leq 5 \operatorname{MinSupp}_{V}(G)$ still holds provided that $|V|$ is large enough.

We close this section by handling the case (2) in Theorem 3.8 In this case $\operatorname{MinSupp}_{V}(G)$ is bounded. (It is 1 and 2 for $G_{0} \simeq S_{m}$ and $G_{0} \simeq A_{m}$, respectively.) Therefore, we give a direct proof for Theorem 1.2 in this case. 
Theorem 3.9. Let $U$ be an m-dimensional vector space over $\mathbb{F}_{q}$, and let $G=S_{m}$ with its natural permutation action on $U$. Assuming that $(|G|,|U|)=1$, we have

$$
P\left(\text { random } \underline{u} \in U^{c} \text { is a base for } G\right)>1-\frac{1}{m^{c-2}} \text { for any } c \geq 3 \text {. }
$$

Hence three random vectors form a base for $G$ with high probability if $m$ is large.

Proof. The $\mathbb{F}_{q} G$-module $V^{c}$ can be naturally identified with $M^{m \times c}(q)$, the space of $m \times c$-matrices over $\mathbb{F}_{q}$. Under this identification, $G$ acts on $M^{m \times c}(q)$ by permuting the rows of each element of $M^{m \times c}(q)$ in a natural way. Hence, a matrix $a \in$ $M^{m \times c}(q)$ is a base for $G$ if and only if the rows of $a$ are pairwise different elements of $M^{1 \times c}(q)$, the space of $c$-dimensional row vectors over $\mathbb{F}_{q}$. Thus, the probability in question is equal to the probability that $m$ random elements of $M^{1 \times c}(q)$ are pairwise different, which is

$$
\prod_{i=0}^{m-1} \frac{q^{c}-i}{q^{c}}>\left(\frac{q^{c}-q}{q^{c}}\right)^{m} \geq\left(1-\frac{1}{m^{c-1}}\right)^{m} \geq 1-\frac{1}{m^{c-2}}
$$

where the first and second inequalities follows since $m<q$ by the coprime assumption. The claim follows.

Corollary 3.10. Let $V$ be an $n$ dimensional vector space over the finite field $\mathbb{F}_{q}$ and let $G=Z \cdot G_{0} \leq G L(V)$ be a coprime linear group, where $G_{0} \simeq S_{m}$ or $G_{0} \simeq A_{m}$ and $V$ is the non-trivial irreducible component of the natural $\mathbb{F}_{q} G_{0}$-module. Then we have

$$
P\left(\text { random } \underline{v} \in V^{c} \text { is a base for } G\right)>1-\frac{1}{n^{c-2}} \text { for any } c \geq 3 .
$$

Proof. First, note that $\operatorname{Fix}(g)=0$ for every $g \in G \backslash G_{0}$, so a $\underline{v} \in V^{c}$ is a base for $G$ if and only if it is a base for $G_{0}$. Second, let $U=V \oplus U_{0}$, where $U_{0}$ is the trivial module for $G_{0}$. For any random vectors $u_{1}, \ldots, u_{c} \in U$ let $v_{i}$ be the projection of $u_{i}$ to $V$ along $U_{0}$. Then $u_{1}, \ldots, u_{c}$ is a base for $G_{0}$ if and only if $v_{1}, \ldots, v_{c}$ is a base for $G_{0}$, so the claim follows from Theorem 3.9.

\section{Proof of Theorem 1.2}

Let $V$ be an $n$-dimensional vector space over the finite field $\mathbb{F}_{q}$ and let $G \leq$ $G L(V)=G L(n, q)$ be a coprime primitive linear group, which is maximal, i.e. there is no coprime subgroup $L \leq G L(V)$ strictly containing $G$. In the following, we give a structure theorem of such groups very similar to a result about maximal solvable primitive linear group (see [21, Lemma 2.2] and [23, §§19-20]). Our proof uses ideas similar to those can be found in [5, [8], and [23]. For the convienience of the reader, we give a self-contained proof here.

In the following, we extend the vector space structure on $V$ by defining multiplication on $V$ with elements from a (possibly) larger field $\mathbb{F}_{q^{k}} \geq \mathbb{F}_{q}$ for some $k \mid n$. In

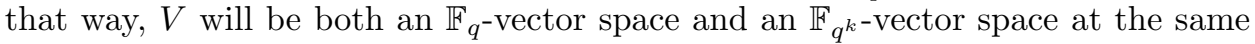
time.

We will use the notation $V=V_{n}(q), V=V_{d}\left(q^{k}\right)$ or $V=V\left(q^{k}\right)$ if we would like to highlight the base field and/or the dimension of $V$.

Theorem 4.1. Let $V=V_{n}(q)$ be an $n$-dimensional vector space over the finite field $\mathbb{F}_{q}$ and let $G \leq G L(V)$ be a maximal coprime primitive linear group. Then the following statements hold. 
(1) There is a unique maximal Abelian subgroup $Z \leq G L(V)$, which is normalised by $G$. Moreover, $Z$ is contained in $G$.

(2) $Z$ is cyclic and $Z \cup\{0\} \simeq \mathbb{F}_{q^{k}}$ for some $k \mid n$.

(3) There is a (unique and maximal) $\mathbb{F}_{q^{k}}$ vector space structure $V=V_{d}\left(q^{k}\right)$ on $V$ for $d=n / k$ such that $\left.G \leq \Gamma L\left(d, q^{k}\right)\right)$.

(4) Let $H:=G \cap G L\left(d, q^{k}\right)$. Then $Z \leq H=C_{G}(Z) \triangleleft G$, furthermore $Z=$ $Z\left(G L\left(d, q^{k}\right)\right)$ is the group of scalar transformations on $V_{d}\left(q^{k}\right)$ and $G / H$ is included into the Galois group $\mathrm{Gal}\left(\mathbb{F}_{q^{k}}, \mathbb{F}_{q}\right)$.

(5) Let $N=F^{*}(H)$ be the generalised Fitting subgroup of $H$. Then $N / Z$ is the socle of $H / Z$. Furthermore, $V_{d}\left(q^{k}\right)$ is an absolutely irreducible $\mathbb{F}_{q^{k}} N$ module.

(6) Let $N_{1}, \ldots, N_{t}$ be the set of minimal normal subgroups of $H$ above $Z$. Then there is an absolutely irreducible $\mathbb{F}_{q^{k}} N_{i}$-module $V_{i}$ for every $i$ such that $V \simeq V_{1} \otimes_{\mathbb{F}_{q^{k}}} \ldots \otimes_{\mathbb{F}_{q^{k}}} V_{t}$. Furthermore, $N=N_{1} \otimes N_{2} \otimes \ldots \otimes N_{t}$ and $H=H_{1} \otimes H_{2} \otimes \ldots \otimes H_{t}$ where $N_{i} \triangleleft H_{i} \leq G L\left(V_{i}\left(q^{k}\right)\right)$ for every $i$.

(7) If $N_{i} / Z$ is Abelian, then $N_{i}=Z R_{i}$ where $R_{i} \leq N_{i}$ is an extraspecial $r_{i}$ group for some prime $r_{i}$ of order $r_{i}^{2 l_{i}+1}$. Furthermore, $\left|N_{i} / Z\right|=r_{i}^{2 l_{i}}$ and $\operatorname{dim}_{\mathbb{F}_{q^{k}}}\left(V_{i}\right)=r_{i}^{l_{i}}$.

(8) If $N_{i} / Z$ is a direct product of $s$ many isomorphic non-Abelian simple groups, then there is a tensor product decomposition $V_{i}=W_{1} \otimes \ldots \otimes W_{s}$ preserved by $N_{i}$. Then $N_{i}=K_{1} \otimes \ldots \otimes K_{s}$ where $K_{i}=S_{i} Z$ for each $i$, and the $S_{i} \leq$ $G L\left(W_{i}\right)$ are isomorphic quasisimple absolutely irreducible groups. Finally, $H_{i}$ permutes the $K_{i}$-s and the $W_{i}$-s in a transitive way.

Proof. Let $A \leq G L(V)$ be any Abelian subgroup normalised by $G$ and $P$ is the (unique) Sylow- $p$ subgroup of $A$ for $p=\operatorname{char}\left(\mathbb{F}_{q}\right)$. Then $P$ is normalised by $G$. Then $0 \neq \operatorname{Fix}_{V}(P)=\cap_{p \in P} \operatorname{Fix}_{V}(p) \leq V$ is $G$-invariant. Since $V$ is an irreducible $\mathbb{F}_{q} G$-module, we get that $P=1$, so $|A|$ is coprime to $|V|$. Therefore, $G A \geq G$ is a coprime linear group, so $A \leq G$ by the maximality of $G$ and part of (1) is proved.

Let $Z \leq G L(V)$ be a maximal Abelian subgroup normalised by $G$. By the previous paragraph, $Z \triangleleft G$. Since $G \leq G L(V)$ is primitive linear, $V$ is a homogeneous $\mathbb{F}_{q} Z$-module. If $V=V_{1} \oplus \ldots \oplus V_{d}$ is a decomposition of $V$ into (isomorphic) irreducible $\mathbb{F}_{q} Z$-modules, then $Z \simeq Z_{V_{i}} \leq \operatorname{End}_{Z}\left(V_{i}\right) \simeq \mathbb{F}_{q^{k}}$ for some $k \geq 1$ by using Schur Lemma. Then $\langle Z\rangle_{\mathbb{F}_{q}}$ (the subalgebra of $\operatorname{End}(V)$ generated by $Z$ ) is isomorphic to the field $\mathbb{F}_{q}^{k}$, and it is invariant under the conjugation by elements of $G$. It follows that $\langle Z\rangle_{\mathbb{F}_{q}} \backslash\{0\} \simeq \mathbb{F}_{q}^{*}$ is an Abelian subgroup of $G L(V)$ normalised by $G$. Therefore, (2) follows by the maximality of $Z$.

Identifying $Z \cup 0 \leq \operatorname{End}(V)$ with $\mathbb{F}_{q}^{k}$, it defines an $\mathbb{F}_{q^{k}}$ vector space structure on $V$. The conjugation action of $G$ on $Z \cup\{0\}=\mathbb{F}_{q^{k}}$ defines a homomorphism $\sigma: G \mapsto \operatorname{Gal}\left(\mathbb{F}_{q^{k}}, \mathbb{F}_{q}\right)$. Now, for any $g \in G, \alpha \in \mathbb{F}_{q}^{k}$ and $v \in V$ we have $g(\alpha v)=$ $\left(g \alpha q^{-1}\right) g(v)=\alpha^{\sigma(g)}(v)$, so $G$ is included into the semilinear group $\Gamma L\left(V_{d}\left(q^{k}\right)\right)=$ $\Gamma L\left(d, q^{k}\right)$. The subgroup $H$ is just the kernel of $\sigma$, so (4) and part of (3) follows.

Let $B \triangleleft G$ be any Abelian normal subgroup, $\alpha \in Z$ a generator of $Z$ and $b \in B$. Then $b \alpha b^{-1}=\alpha^{\sigma(b)}=\alpha^{q^{s}}$ for some $0 \leq s<k$, so $[b, \alpha]=\alpha^{q^{s}-1} \in B$ is centralised by $b$. Changing $b$ to $b^{-1}$ if necessary, we can assume that $0 \leq s \leq k / 2$. This means $\left(\alpha^{q^{s}-1}\right)^{q^{s}}=\alpha^{q^{s}-1}$, so $q^{k}-1 \mid\left(q^{s}-1\right)^{2}<q^{k}-1$. Therefore, $s=0$. Thus, $B \leq C_{G}(Z)$, so $B Z \geq Z$ is an Abelian normal subgroup in $G$. By the maximality of $Z$, we get $B \leq Z$, which completes the proof of both (1) and (3). 
Let $M=F(H)$ be the Fitting subgroup of $H$. Then $Z(M)$ is an Abelian normal subgroup of $G$, so $Z(M)=Z$ by the maximality of $Z$. Let $n$ by the nilpotency class of $M$. If $n=1$ then $M=Z$. Otherwise, we claim that $n=2$. Assuming that $n \geq 3$, we have $1 \neq \gamma_{n}(M) \leq Z$, and $\left[\gamma_{n-1}(M), \gamma_{n-1}(M)\right] \leq\left[\gamma_{2}(M), \gamma_{n-1}(M)\right] \leq$ $\gamma_{n+1}(M)=1$, so $\gamma_{n-1}(M)$ is an Abelian normal subgroup of $G$, so it must contained in $Z$. This forces $\gamma_{n}(M)=1$, a contradiction. Therefore, $n \leq 2$, that is, $M / Z$ is Abelian.

Let $R$ be a Sylow- $r$-subgroup of $M$ for some prime $r$ dividing $|M / Z|$. The commutator map defines a symplectic bilinear function from $R / Z$ into $Z(R)=$ $R \cap Z$. Therefore, for any $x, y \in R$ we have $\left[x^{r}, y^{r}\right]=[x, y]^{r^{2}}=\left[x^{r^{2}}, y\right]$. If $r^{s}$ is the exponent of $R /(R \cap Z)$ for some $s \geq 2$, then $R^{r^{s-1}} Z$ is an Abelian normal subgroup of $G$, so $R^{r^{s-1}} \leq Z$, a contradiction. Thus, we get $R /(R \cap Z)$ is an elementary Abelian $r$-group. Using this and the above commutator identity it also follows that $R^{\prime} \leq Z$ is of exponent $r$. It follows that $R=(R \cap Z) R_{0}$ for some extraspecial $r$-group $R_{0}$.

Be the previous two paragraphs, $F(H) / Z$ is exactly the direct product of the minimal Abelian normal subgroups of $H$, so $F(H) / Z$ is contained in $\operatorname{Soc}(H / Z)$. Since $N=F^{*}(H)$ is the central product of $F(H)$ and the layer $E(H)$, where $E(H) / Z$ is the direct product of the minimal non-Abelian normal subgroups of $H / Z$ it follows that $N / Z=\operatorname{Soc}(H / Z)$ as claimed. By [5, Lemma 12.1], $V_{d}\left(q^{k}\right)$ is an absolutely irreducible $\mathbb{F}_{q^{k}} H$-module. If the irreducible $\mathbb{F}_{q^{k}} N$-components of $V_{d}\left(q^{k}\right)$ were not be absolutely irreducible, then $Z\left(C_{G L\left(V_{d}\left(q^{k}\right)\right)}(N)\right)$ would be the multiplicative group of a proper field extension of $\mathbb{F}_{q^{k}}$ normalised by $G$, which again contradicts with the maximility of $Z$. Now, let us assume that $V_{d}\left(q^{k}\right)=$ $U \oplus \ldots \oplus U$ is a direct sum of $s$ many isomorphic absolutely irreducible $\mathbb{F}_{q^{k}} N$ modules for some $s \geq 2$. By [12, Lemma 4.4.3(ii)], there is a tensor product decomposition $U \otimes_{\mathbb{F}_{q^{k}}} \bar{W}$ of $V_{d}\left(q^{k}\right)$ such that $N \leq G L(U) \otimes 1_{W} \leq G L(U) \otimes G L(W)$ and $G \leq N_{\Gamma L(V)}(N) \leq N_{\Gamma L(V)}(G L(U) \otimes G L(W))$. Let $L=\left\{1_{U} \otimes h_{W} \mid \exists h_{U} \in\right.$ $G L(U)$ such that $\left.h_{U} \otimes h_{W} \in H\right\}$. If $L=Z$, then $V_{d}\left(q^{k}\right)$ is not irreducible as an $\mathbb{F}_{q^{k}} H$-module, a contradiction. We have $L \leq G L(V)$ is a coprime linear group normalised by $G$, so $L G \leq G L(V)$ is coprime. Using the maximality of $G$ we get that $L<G$. But then $Z<L \leq H$ clearly centralises $N=F^{*}(H)$, a contradiction. So, $V_{d}\left(q^{k}\right)$ is an absolutely irreducible $\mathbb{F}_{q^{k}} N$-module, and (5) is proved. Now, (6) follows by a combined use of [16, Corollary 18.2/(a)] and [12, Lemma 4.4.3(iii)].

If $N_{i} / Z$ is Abelian, then it is a minimal Abelian normal subgroup of $H / Z$ so it is elementary Abelian $r_{i}$-group for some prime $r_{i}$. Using the same argument as in paragraph 6 of this proof, one can find the extraspecial $r_{i}$-group $R_{i}$ by taking the full inverse image of a maximal non-degenerate subspace of $R / R^{\prime}$ where $R$ is the Sylow$r_{i}$ subgroup of $N_{i}$. For this subgroup, it clearly follows that $N_{i}=Z R_{i}$, and $\left|R_{i}\right|=$ $r_{i}^{2 l_{i}+1}$ for some integer. Furthermore, since $V_{i}$ is an absolutely irreducible $\mathbb{F}_{q^{k}} N_{i^{-}}$ module, it must be an absolutely irreducible $\mathbb{F}_{q^{k}} R_{i}$-module. It is well-known that extraspecial $r_{i}$-group of order $r_{i}^{2 l_{i}+1}$ has a unique faithfull absolutely irreducible ordinary representation, and this representation has degree $r_{i}^{l_{i}}$, which finishes the proof of $(7)$.

Finally, (8) can again be deduced from [16, Corollary 18.2/(a)] and from the fact that $N_{i} / Z$ is a minimal normal subgroup in $H_{i} / Z$. 
Lemma 4.2. Let $G$ be a group, $K$ be a field and let $V$ be an arbitrary finite dimensional $K G$-module.

(1) For any $g, h \in G$ we have $\operatorname{Supp}([g, h]) \leq 2 \operatorname{Supp}(g)$.

(2) If $N \triangleleft G$ such that $V$ is absolutely irreducible as a $K N$-module, then $\operatorname{MinSupp}(N) \leq 2 \operatorname{MinSupp}(G)$.

Proof. Let us consider the subspaces $U=\operatorname{Fix}(g)$ and $W=\operatorname{Fix}\left(h^{-1} g h\right)$ of $V$. Then we have

$$
\operatorname{dim}(U)+\operatorname{dim}(W)-\operatorname{dim}(U \cap W)=\operatorname{dim}(U+W) \leq \operatorname{dim}(V) .
$$

Using that $\operatorname{dim}(U)=\operatorname{dim}(W)=\operatorname{dim}(V)-\operatorname{Supp}(g)$ we get $\operatorname{dim}(U \cap W) \geq \operatorname{dim}(V)-$ $2 \operatorname{Supp}(g)$. On the other hand $U \cap W \leq \operatorname{Fix}([g, h])$ holds trivially, so

$$
\begin{aligned}
\operatorname{Supp}([g, h]) & =\operatorname{dim}(V)-\operatorname{dim}(\operatorname{Fix}([g, h])) \leq \operatorname{dim}(V)-\operatorname{dim}(U \cap W) \\
& \leq \operatorname{dim}(V)-(\operatorname{dim}(V)-2 \operatorname{Supp}(g))=2 \operatorname{Supp}(g),
\end{aligned}
$$

and part (1) follows.

For part (2), let $1 \neq g \in G$ be any element. If $[g, N]=1$, then $g$ acts as a scalar transformation on $V$ by [10, Theorem 9.2], so $\operatorname{Supp}(g)=\operatorname{dim}(V) \geq \operatorname{MinSupp}(N)$. Otherwise, there is an element $n \in N$ such that $[g, n] \neq 1$. Then we have $\operatorname{MinSupp}(N) \leq \operatorname{Supp}([g, n]) \leq 2 \operatorname{Supp}(g)$. Thus, $\operatorname{MinSupp}(N) \leq 2 \operatorname{Supp}(g)$ for every $1 \neq g \in G$, which proves that $\operatorname{MinSupp}(N) \leq 2 \operatorname{MinSupp}(G)$.

Lemma 4.3. Let $V_{1}, \ldots, V_{k}$ be finite dimensional vector spaces over the field $\mathbb{F}_{q}$ and $Z<G_{1} \leq G L\left(V_{1}\right), \ldots, Z<G_{k} \leq G L\left(V_{k}\right)$ be coprime linear groups. Consider the group $G:=G_{1} \otimes \ldots \otimes G_{k}$ acting on the tensor product $V:=V_{1} \otimes \ldots \otimes V_{k}$ in a natural way.

(1) Let $g=g_{1} \otimes \ldots \otimes g_{k} \in G$ with $g_{j} \in G_{j}$ for each $j$ and let us assume that $g_{i} \notin Z$ for some $i$. Then

$$
\operatorname{Supp}_{V}(g) \geq \operatorname{MinSupp}_{V_{i}}\left(G_{i}\right) \cdot \frac{\operatorname{dim}(V)}{\operatorname{dim}\left(V_{i}\right)}
$$

or $\operatorname{Supp}_{V}(g) \geq \frac{1}{2} \operatorname{dim}(V)$.

(2) As a consequence

$$
\begin{aligned}
& \quad \operatorname{MinSupp}_{V}(G)=\min _{i}\left\{\operatorname{MinSupp}_{V_{i}}\left(G_{i}\right) \cdot \frac{\operatorname{dim}(V)}{\operatorname{dim}\left(V_{i}\right)}\right\}, \\
& \text { or } \operatorname{MinSupp}_{V}(G) \geq \frac{1}{2} \operatorname{dim}(V) \text {. }
\end{aligned}
$$

Proof. To prove part (1), first we consider the case $k=2$. Let $n_{1}=\operatorname{dim}\left(V_{1}\right), n_{2}=$ $\operatorname{dim}\left(V_{2}\right)$, so $n=\operatorname{dim}(V)=n_{1} n_{2}$. Furthermore, let $1 \neq g=g_{1} \otimes g_{2} \in G_{1} \otimes G_{2}$ be an element of $G$ with $g_{1} \notin Z$. Since the action is coprime, $g_{1}$ and $g_{2}$ are diagonalisable over $\overline{\mathbb{F}}_{q}$. Let $\alpha_{1}, \ldots \alpha_{s} \in \overline{\mathbb{F}}_{q}$ be the different eigenvalues of $g_{1}$ with multiplicity $k_{1}, k_{2}, \ldots, k_{s}$. We can assume that $k_{1}$ is the largest among the $k_{i}$. Let $l_{1}, \ldots, l_{s}$ be the multiplicities of $\alpha_{1}^{-1}, \ldots, \alpha_{s}^{-1}$ in the characteristic polynomial of $g_{2}$ (Some of them can be zero). Then

$$
\begin{aligned}
\operatorname{Supp}_{V}(g) & =\operatorname{Supp}_{V}\left(g_{1} \otimes g_{2}\right)=n-\operatorname{dim}\left(\operatorname{Fix}_{V}\left(g_{1} \otimes g_{2}\right)\right) \\
& =n-\sum_{i=1}^{s} k_{i} l_{i} \geq n-\sum_{i=1}^{s} k_{1} l_{i} \geq\left(n_{1}-k_{1}\right) n_{2} .
\end{aligned}
$$


If $\alpha_{1} \in \mathbb{F}_{q}$, then we can substitute $g_{1}$ by $\alpha_{1}^{-1} g_{1}$ and $g_{2}$ by $\alpha_{1} g_{2}$ (since both $G_{1}$ and $G_{2}$ contains all the scalar transformations), so we can assume that $\alpha_{1}=1$. Now, since $g_{1} \neq 1$, we get $\operatorname{Supp}_{V}(g) \geq\left(n_{1}-k_{1}\right) n_{2}=\operatorname{Supp}_{V_{1}}\left(g_{1}\right) n_{2} \geq \operatorname{MinSupp}_{V_{1}}\left(G_{1}\right) \cdot \operatorname{dim}\left(V_{2}\right)$.

Now, let us assume that $\alpha_{1} \notin \mathbb{F}_{q}$. Then there is an algebraic conjugate element of $\alpha_{1}$ (different from $\alpha_{1}$ ) under the action of $\operatorname{Gal}\left(\overline{\mathbb{F}}_{q}, \mathbb{F}_{q}\right)$ which is also an eigenvalue of $g_{1}$ with the same multiplicty as $\alpha_{1}$. In particular, $k_{1} \leq n_{1} / 2$. Thus,

$$
\operatorname{Supp}_{V}(g) \geq\left(n_{1}-k_{1}\right) n_{2} \geq\left(n_{1} / 2\right) n_{2}=\frac{\operatorname{dim}(V)}{2} .
$$

By changing the role of $g_{1}$ and $g_{2}$ in the proof and by using induction on $k$, we get the claim of part (1).

Finally, if $\operatorname{Supp}_{V_{i}}\left(g_{i}\right)=\operatorname{MinSupp}_{V_{i}}\left(G_{i}\right)$ for some $g_{i} \notin Z$, then

$$
\operatorname{Supp}_{V}\left(1 \otimes \ldots \otimes 1 \otimes g_{i} \otimes 1 \otimes \ldots \otimes 1\right)=\operatorname{MinSupp}_{V_{i}}\left(G_{i}\right) \cdot \frac{\operatorname{dim}(V)}{\operatorname{dim}\left(V_{i}\right)},
$$

so part (2) follows by using part (1).

Proof of Theorem 1.2. Let $G \leq G L(V)$ be a coprime primitive linear group. Without loss of generality we can assume that $G$ is maximal among such subgroups of $G L(V)$. Let $Z$ be the unique maximal Abelian subgroup in $G L(V)$ which is normalised by $G$ and $H$ be the intersection of $G$ and $G L\left(d, q^{k}\right)$ as in Theorem 4.1. If $g \in G \backslash H$ then there is a $z \in Z$ such that $[g, z] \neq 1$. By Lemma 4.2, $\operatorname{Supp}_{V}(g) \geq \frac{1}{2} \operatorname{Supp}_{V}([g, z])=\frac{1}{2} \operatorname{dim}(V)$. Therefore if $c>4$, then

$$
\sum_{g \in G \backslash H} \frac{1}{q^{c \cdot \operatorname{Supp}_{V}(g)}} \leq \frac{|G \backslash H|}{q^{\frac{c}{2} \operatorname{dim}(V)}} \leq \frac{|V|^{2}}{|V|^{\frac{c}{2}}} \leq \frac{1}{|V|^{\frac{c}{2}-2}} .
$$

Now let $g$ be an element of $H=H_{1} \otimes \ldots \otimes H_{t}$. So $g=\left(g_{1}, \ldots, g_{t}\right)$ where $g_{i} \in H_{i}$ for all $i \in[t]$ and $g$ preserves the tensor product decomposition $V=$ $V_{1} \otimes \ldots \otimes V_{t}$ over $\mathbb{F}_{q}^{k}$ as in Theorem 4.1 (6) and $\operatorname{dim}_{\mathbb{F}_{q^{k}}}\left(V_{i}\right)=d_{i}$ for all $i$ (therefore $d=\operatorname{dim}_{\mathbb{F}_{q^{k}}}(V)=\prod_{i=1}^{t} d_{i}$ and $\left.\operatorname{dim}(V)=\operatorname{dim}_{\mathbb{F}_{q}}(V)=k \cdot \prod_{i=1}^{t} d_{i}\right)$. We can assume that in this decomposition the dimensions of the vector spaces are decreasing, i.e. $d_{1} \geq d_{2} \geq \ldots \geq d_{t} \geq 2$. Let $g_{i} \notin Z$ for some $i \neq 1$. Then by Lemma 4.3 ,

$$
\operatorname{Supp}_{V}(g) \geq \operatorname{MinSupp}_{V_{i}}\left(H_{i}\right) \cdot \frac{\operatorname{dim}(V)}{\operatorname{dim}\left(V_{i}\right)} \geq k \prod_{j \neq i} d_{j} \text {. }
$$

Since $2 \prod_{j \neq i} d_{j} \geq 2^{t-1} d_{1} \geq \sum_{i=1}^{t} d_{1}$ and $k \prod_{j \neq i} d_{j} \geq k \sqrt{\prod_{j=1}^{t} d_{j}} \geq \sqrt{\operatorname{dim}(V)}$ we get that

$$
c \cdot \operatorname{Supp}_{V}(g) \geq 2 k \sum_{i=1}^{t} d_{i}+(c-4) \sqrt{\operatorname{dim}(V)} .
$$

Hence,

$$
\begin{aligned}
\sum_{g \in H \backslash H_{1}} \frac{1}{q^{c \cdot \operatorname{Supp}_{V}(g)}} & \leq \frac{\prod_{i=1}^{t}\left|H_{i}\right|}{q^{2 k \sum_{i=1}^{t} d_{i}+(c-4) \sqrt{\operatorname{dim}(V)}}} \\
& \leq \frac{q^{2 k \sum_{i=1}^{t} d_{i}}}{q^{2 k \sum_{i=1}^{t} d_{i}+(c-4) \sqrt{\operatorname{dim}(V)}}}=\frac{1}{q^{(c-4) \sqrt{\operatorname{dim}(V)}}}
\end{aligned}
$$


Now assume that $g \in H_{1}$. In this case $\operatorname{Supp}_{V}(g)=\operatorname{Supp}_{V_{1}}(g) \cdot \frac{d}{d_{1}}$. By Theorem 4.1. $Z \leq N_{1} \leq H_{1} \leq G L\left(V_{1}\left(q^{k}\right)\right)$ where $N_{1}$ is a minimal normal subgroup above $Z$ and $N_{1} / Z$ is characteristically simple. Therefore, it is either an elementary Abelian group, a direct product of non-Abelian simple groups, or a non-Abelian simple group.

First, if $N_{1} / Z$ is elementary Abelian, then $N_{1}=Z \cdot P$ where $P$ is an extraspecial $r$-group for a prime $r$ with $r \mid q^{k}-1$. Then $V_{1}\left(q^{k}\right)$ is an absolutely irreducible $\mathbb{F}_{q^{k}} P$-module. If $n \in P \backslash Z$ then $n$ has exactly $r$ different eigenvalues on $V_{1}$ (or on $V)$ each with the same multiplicity. It follows that $\operatorname{MinSupp}_{V}\left(N_{1}\right) \geq \frac{r-1}{r} \operatorname{dim}(V) \geq$ $\frac{1}{2} \operatorname{dim}(V)$, so $\operatorname{MinSupp}_{V}\left(H_{1}\right) \geq \frac{1}{4} \operatorname{dim}(V)$ by Lemma 4.2. In this case,

$$
\sum_{g \in H_{1}} \frac{1}{q^{c \cdot \operatorname{Supp}_{V}(g)}} \leq \frac{\left|H_{1}\right|}{q^{\frac{c}{4} \operatorname{dim}(V)}} \leq \frac{|V|^{2}}{q^{2 \operatorname{dim}(V)+\left(\frac{c}{4}-2\right) \operatorname{dim}(V)}} \leq \frac{1}{|V|^{\frac{c}{4}-2}} .
$$

Next, let $N_{1} / Z$ is a direct product of $s \geq 2$ many isomorphic non-Abelian simple groups. By Theorem 4.1 (8), the action of $N_{1}=K_{1} \otimes \ldots \otimes K_{s}$ on $V_{1}$ preserves a tensor product decomposition $V_{1}=W_{1} \otimes \ldots \otimes W_{s}$ over $\mathbb{F}_{q}^{k}$, where $\operatorname{dim}_{\mathbb{F}_{q^{k}}}\left(W_{i}\right)=$ $\sqrt[s]{d_{1}} \geq 2$ for every $i$. Using [17, Theorem 1 ], we get that

$$
\left|N_{1}\right| \leq \prod_{i=1}^{s}\left|K_{s}\right| \leq \prod_{i=1}^{s}\left|W_{i}\right|^{2}=q^{2 k s \sqrt[s]{d_{1}}} .
$$

On the other hand, $H_{1} / N_{1}$ acts faithfully on $\left\{W_{1}, \ldots, W_{s}\right\}$ and $\left|H_{1} / N_{1}\right|$ is coprime to $q$, so $\left|H_{1} / N_{1}\right| \leq q^{s}$ by [8, Corollary 2.4]. Therefore, $\left|H_{1}\right| \leq q^{2 k s} \sqrt[s]{d_{1}}+s$. By Lemma 4.2 and by Lemma 4.3 .

$$
\operatorname{Supp}_{V_{1}}(g) \geq \frac{1}{2} \operatorname{MinSupp}_{V_{1}}\left(N_{1}\right) \geq \frac{k}{2} \cdot \frac{d_{1}}{\sqrt[s]{d_{1}}} .
$$

Therefore,

$c \operatorname{Supp}_{V}(g) \geq 5 k d_{1}{ }^{(s-1) / s}+\left(\frac{c}{2}-5\right) k \sqrt{d_{1}} \cdot \frac{d}{d_{1}} \geq 2 k s \sqrt[s]{d_{1}}+s+\left(\frac{c}{2}-5\right) \sqrt{\operatorname{dim}(V)}$.

So,

$$
\begin{aligned}
\sum_{1 \neq g \in H_{1}} \frac{1}{q^{c \cdot \operatorname{Supp}_{V}(g)}} & \leq \frac{\left|H_{1}\right|}{q^{c \cdot \operatorname{MinSupp}_{V}\left(H_{1}\right)}} \leq \frac{q^{2 k s} \sqrt[s]{d_{1}+s}}{q^{2 k s \sqrt[s]{d_{1}}+s+\left(\frac{c}{2}-5\right) \sqrt{\operatorname{dim}(V)}}} \\
& \leq \frac{1}{q^{\left(\frac{c}{2}-5\right) \sqrt{\operatorname{dim}(V)}}} .
\end{aligned}
$$

Finally, let $N_{1} / Z$ be a non-Abelian simple group. If $d_{1} \leq \sqrt{d}$, then we can use the same argument as in the previous paragraph to get that

$$
\sum_{1 \neq g \in H_{1}} \frac{1}{q^{c \cdot \operatorname{Supp}_{V}(g)}} \leq \frac{1}{q^{(c-2) \sqrt{\operatorname{dim}(V)}}} .
$$

Summarizing the bounds given until this point, we get that

$$
\begin{aligned}
P b(c, G, V) & \geq 1-\sum_{1 \neq g \in G} \frac{1}{q^{c \cdot \operatorname{Supp}_{V}(g)}} \geq 1-\left(\frac{1}{|V|^{\frac{c}{2}-2}}+\frac{1}{q^{(c-4) \sqrt{\operatorname{dim}(V)}}}\right. \\
& \left.+\frac{1}{q^{\left(\frac{c}{2}-5\right)} \sqrt{\operatorname{dim}(V)}}\right) \geq 1-\frac{3}{q^{\left(\frac{c}{2}-5\right) \sqrt{\operatorname{dim}(V)}}},
\end{aligned}
$$


which is case (1) of Theorem 1.2

Now, let us assume that $d_{1} \geq \sqrt{d}$. If $\left|V_{1}\right|=q^{k d_{1}}$ is bounded by the constant appearing in part (3) of Theorem [3.8, then $|V|$ is also bounded. Hence we can assume that either part (1) or part (2) of Theorem 3.8 holds. By Lemma 4.2, we also have $\operatorname{MinSupp}_{V}\left(H_{1}\right) \geq \frac{1}{2} \operatorname{MinSupp}_{V}\left(N_{1}\right)$.

If $N_{1} / Z$ is not an alternating group, then $\operatorname{MinSupp}_{V}\left(N_{1}\right) \geq \frac{1}{40} \operatorname{dim}(V)$ and $5 \cdot \operatorname{MinSupp}_{V}\left(N_{1}\right) \geq \log _{q}\left|H_{1}\right|$ by using Corollary 3.3. Theorem 3.8/(1) and Lemma $4.3 /(2)$. Thus, we have

$$
\sum_{1 \neq g \in H_{1}} \frac{1}{q^{c \cdot \operatorname{Supp}_{V}(g)}} \leq \frac{1}{|V|^{(c-10) / 80}} .
$$

So, in this case we get that

$$
\begin{aligned}
P b(c, G, V) & \geq 1-\left(\frac{1}{|V|^{\frac{c}{2}-2}}+\frac{1}{q^{(c-4)} \sqrt{\operatorname{dim}(V)}}+\frac{1}{|V|^{(c-10) / 80}}\right) \\
& \geq 1-\left(\frac{1}{q^{(c-4) \sqrt{\operatorname{dim}(V)}}}+\frac{2}{|V|^{(c-10) / 80}}\right),
\end{aligned}
$$

which is case (2)/a of Theorem 1.2 .

Finally, let $N_{1} / Z \simeq A_{m}$ for some $m$. If $V_{1}$ is not an irreducible component of the natural $\mathbb{F}_{q}^{k} A_{m}$ permutation module, then we have $\operatorname{MinSupp}_{V}\left(N_{1}\right) \geq \frac{1}{16} \sqrt{\operatorname{dim}(V)}$ and $5 \cdot \operatorname{MinSupp}_{V}\left(N_{1}\right) \geq \log _{q}\left|H_{1}\right|$ by using Corollary 3.7. Theorem 3.8/(1) and Lemma 4.3/ (2). Thus, we have

$$
\sum_{1 \neq g \in H_{1}} \frac{1}{q^{c \cdot \operatorname{Supp}_{V}(g)}} \leq \frac{1}{q^{\frac{c-10}{16}} \sqrt{\operatorname{dim}(V)}}
$$

and

$$
\begin{aligned}
P b(c, G, V) & \geq 1-\left(\frac{1}{|V|^{\frac{c}{2}-2}}+\frac{1}{q^{(c-4)} \sqrt{\operatorname{dim}(V)}}+\frac{1}{q^{\frac{c-10}{16}} \sqrt{\operatorname{dim}(V)}}\right) \\
& \geq 1-\frac{3}{q^{\frac{c-10}{16} \sqrt{\operatorname{dim}(V)}}} .
\end{aligned}
$$

Finally, if $V_{1}$ is the non-trivial irreducible component of the natural $\mathbb{F}_{q}^{k} A_{m}$-module, then with the use of Corollary 3.10 we get that

$$
P b(c, G, V) \geq 1-\left(\frac{1}{|V|^{\frac{c}{2}-2}}+\frac{1}{q^{(c-4)} \sqrt{\operatorname{dim}(V)}}+1-P b\left(c, H_{1}, V\right)\right) \geq 1-\frac{3}{n^{c-2}},
$$

which completes the proof of Theorem 1.2

\section{REFERENCES}

[1] T. C. Burness, M. W. Liebeck, A. Shalev, Base sizes for simple groups and a conjecture of Cameron, Proc. Lond. Math. Soc. (3) 98 (1) (2009) 116-162.

[2] P. J. Cameron, W. M. Kantor, Random permutations: some group-theoretic aspects, Combin. Probab. Comput. 2 (3) (1993) 257-262.

[3] P. J. Cameron, Permutation Groups, London Mathematical Society Student Texts, vol.45, Cambridge University Press, Cambridge, 1999

[4] The GAP Group, Groups, Algorithms, and Programming, 2014.

[5] M. Giudici, M. W. Liebeck, C. E. Praeger, J. Saxl, P. H. Tiep, Arithmetic results on orbits of linear groups, Trans. Amer. Math. Soc. 368 (2014), 2415-2467.

[6] D. Gluck, Sharper character value estimates for groups of Lie type J. Algebra 174 (1995), 229-266. 
[7] D. Gluck, K. Magaard, Base sizes and regular orbits for coprime affine permutation groups, J. Lond. Math. Soc. (2) 58 (1998) 603618.

[8] Z. Halasi and K. Podoski, Every coprime linear group admits a base of size two, Trans. Amer. Math. Soc. 368 (2016), 5857-5887.

[9] P. N. Hoffman and J. F. Humphreys, Projective representations of the symmetric groups. $Q$ functions and shifted tableaux. Oxford Mathematical Monographs. Oxford University Press, New York (1992)

[10] I. M. Isaacs, Character theory of finite groups, Pure and Applied Mathematics, No. 69. Academic Press, New York-London, 1976.

[11] G. D. James, The representation theory of the symmetric groups. Lect. Notes Math., vol. 682. Springer, Berlin (1978)

[12] P. Kleidman and M. W. Liebeck, The Subgroup Structure of the Finite Classical Groups, London Math. Soc. Lecture Note Series, Vol. 129, Cambridge University Press, 1990.

[13] A. S. Kleshchev and P. H. Tiep, Small-dimensional projective representations of symmetric and alternating groups, Algebra Number Theory 6 (2012), 1773-1816.

[14] M. W. Liebeck, Character ratios for finite groups of Lie type, and applications, Contemp. Math., 694, Amer. Math. Soc., (2017), 193-208.

[15] M. W. Liebeck, A. Shalev, Simple groups, permutation groups, and probability, J. Amer. Math. Soc. 12(2) (1999) 497-520.

[16] G. Malle and D. Testerman, Linear algebraic groups and finite groups of Lie type, Cambridge Studies in Advanced Mathematics, vol. 133, Cambridge University Press, Cambridge, 2011.

[17] P. P. Pálfy and L. Pyber, Small groups of automorphisms, Bull. London Math. Soc. 30 (1998), 386-390.

[18] L. Pyber, Asymptotic results for permutation groups, in: L. Finkelstein, W. M. Kantor (Eds.), Groups and Computation, in: DIMACS Ser. Discrete Math. Theoret. Comput. Sci., vol.11, Amer. Math. Soc., Providence, RI, 1993, pp.197-219.

[19] L. Pyber, personal communication, Bielefeld, 2017.

[20] R. Rasala, On the minimal degrees of characters of $S_{n}$, J. Algebra 45 (1977), 132-181.

[21] Á. Seress, The minimal base size of primitive solvable permutation groups, J. Lond. Math. Soc. 53 (1996), 243-255.

[22] Á. Seress, Permutation Group Algorithms, Cambridge Tracts in Mathematics, vol.152, Cambridge University Press, Cambridge, 2003.

[23] D. A. Suprunenko, Matrix groups, Amer. Math. Soc., Providence, RI, 1976.

Department of Mathematics, Central European University, Nádor utca 9., H-1051, BudAPEST, Hungary

E-mail address: duyan_hulya@phd.ceu.edu

Department of Algebra and Number Theory, EÖtvös University, Pázmány Péter SÉtÁny 1/C, H-1117, Budapest, Hungary and Alfréd Rényi Institute of Mathematics, Hungarian Academy of Sciences, Reáltanoda utca 13-15, H-1053, Budapest, Hungary, ORCID: HTTPS://ORCID. ORG/0000-0002-1305-5380

E-mail address: zhalasi@cs.elte.hu and halasi.zoltan@renyi.mta.hu

Alfréd Rényi Institute of Mathematics, Hungarian Academy of Sciences, Reáltanoda utCa 13-15, H-1053, Budapest, Hungary

E-mail address: podoski.karoly@renyi.mta.hu 